\title{
Severe cognitive impairment in DMD: obvious clinical indication for Dp71 isoform point mutation screening
}

\author{
Marie-Pierre Moizard ${ }^{1}$, Annick Toutain ${ }^{1}$, Delphine Fournier ${ }^{1}$, Françoise Berret ${ }^{1}$, \\ Martine Raynaud $^{1}$, Catherine Billard ${ }^{2}$, Christian Andres ${ }^{3}$ and Claude Moraine ${ }^{1}$
}

\author{
${ }^{1}$ Unité de Génétique, Hôpital Bretonneau; ${ }^{2}$ Unité de N eurochirurgie-N eurologie, Hôpital Clocheville; ${ }^{3}$ Laboratoire de \\ Biochimie et Biologie Moléculaire, INSERM unité316, Faculté de M édecine, Tours, France
}

\begin{abstract}
Duchenne muscular dystrophy is associated with variable degrees of selective cognitive defect with lower scores for verbal intelligence and reading abilities. A number of findings have shown that rearrangements located in the second part of the gene seem to be preferentially associated with cognitive impairment. Several dystrophin transcripts are expressed in the brain. The more distal of them, Dp71, is predominant. We have carried out a mutational analysis of Dp 71 transcript in 12 DMD patients severely, mildly or not retarded, all without detectable deletion or duplication. We have detected five point mutations causing Dp 71 premature translation termination. All were found among the more severely mentally retarded patients of this group (VIQ $<\mathbf{5 0}$ and/or no reading acquisition). European Journal of Human Genetics (2000) 8, 552-556.
\end{abstract}

Keywords: Duchenne muscular dystrophy; Dp71; cognitive impairment; point mutations; dystrophin isoforms

\section{Introduction}

Duchenne muscular dystrophy (DMD) is a progressive $X$-linked muscle degenerative disorder, caused in most cases by large out-of-frame deletions or duplications in the dystrophin gene. ${ }^{1}$ The remaining patients have more subtle mutations such as small insertions/deletions or nucleotide substitutions. ${ }^{2}$ In both cases, mutations cause premature translation termination leading to an absence of dystrophin, a $427 \mathrm{kDa}$ protein that is localised in the plasma membrane of musclecells. Apart from the muscles, the brain is the major site of dystrophin expression. Two $427 \mathrm{kDa}$ dystrophins, one active in neuronal cells of the cerebral cortex and the other expressed in cerebellar Purkinje neurons, originate from two distinct proximal promoters located in the vicinity of the muscle promoter. ${ }^{3,4}$ Two smaller alternative C-terminal products expressed in the brain, Dp140 and Dp71, have also been described. Dp140 is initiated upstream from exon $45^{5}$ and Dp 71, between exons62 and 63.,7 These four DMD transcripts expressed in the brain retain the cysteine-rich and carboxyterminal domains of dystrophin which bind to a

Correspondence: Marie-Pierre Moizard, Unité de Génétique, Hôpital Bretonneau, 2 boulevard Tonnellé, 37044 Tours Cedex, France. Tel:

+33247474799; Fax: + 332476182 56; E-mail: moizard - m@lemel.fr Received 23 July 1999; revised 25 February 2000; accepted

1 March 2000 complex of sarcolemmal proteins known as the dystrophin associated proteins (DAPs). ${ }^{8}$

In most patients, the muscle disease is associated with variable degrees of cognitive impairment, corresponding to significantly lower scores for verbal skills and delay in reading learning. ${ }^{9}$ Several investigations have shown that rearrangements located in the second part of the genetend to be more commonly associated with cognitive impairment than mutations located in the proximal part. ${ }^{10-12}$ Recently Bardoni et $\mathrm{al}^{13}$ found a statistically significant correlation between the absence of Dp140 promoter and the presence of mental retardation in patients suffering from Becker muscular dystrophy (BMD), the allelic milder form of DMD. Moreover, many point mutations have been described in mentally retarded patients in the distal part of the gene corresponding to the Dp71 coding region. ${ }^{14,15}$ In a recent investigation, we found two promoter deleted Dp140 transcripts and two altered Dp71 transcripts (total absence of Dp71 transcript for one patient and a nonsense mutation for the other) respectively in four patients with severe cerebral dysfunction. ${ }^{16}$ Both patients with Dp140 deletion had a QIV $<70$ and bad or no reading acquisition, whereas both patients with altered Dp 71 transcripts were psychologically untestable because of severe mental deficiency. Taken 
together, these findings suggest that: (i) the cognitive impairment in some DMD patients may be related to dysfunction of certain brain DMD isoforms, and (ii) the degree of mental retardation might be related to the location of the mutation in the gene. It seems that the degree of cognitive impairment is more severe when the mutation is more distal.

To support this last hypothesis, we screened for point mutations in the Dp71 coding sequence in a group of 12DMD patients without detectable deletion or duplication in the whole dystrophin cDNA sequence. Seven of the 12 patients were included in our previous study ${ }^{16}$ but were not screened for Dp71 point mutations (patients 7, 11, 13, 22, $43,44,48$ ) (Table 1). We report here four nonsen se mutations and one splice mutation that were all detected in five severely neuropsychologically impaired patients. Results of semiquantitative analysis performed to compare Dp71 transcript amount in mentally retarded patients, some with nonsense mutations and other with no Dp71 mutation, are also reported.

\section{Materials and methods \\ Patients}

After informed consent, all 12 patients were diagnosed on clinical features, progression of the disease, family history, markedly raised serum creatine kinase level and, when performed, absence of dystrophin on muscle biopsy.

When patients could be tested, evaluation of cognitive abilities included verbal (VIQ) and visuospatial (PIQ) intelligence assessment (WISC-R scale) and/or reading skills assessment (Alouette test), as previously described. ${ }^{16}$

Deletion and duplications screening had been performed both by multiplex PCR assays and Southern blotting covering the whole cDNA dystrophin sequence. ${ }^{16}$

\section{Dp 71 transcript analysis}

Dp71 transcript analysis was performed on total RNA isolated from lymphoblastoid cells. In brief, $5 \mu \mathrm{g}$ of total RNA were reverse transcribed into cDNA using $100 \mathrm{pmol}$ of random hexamers and $200 \mathrm{U}$ of Superscript reverse transcriptase (Life Technologies, Inchinnan, UK) in $200 \mu$ l of buffer containing $1 \mathrm{~mm}$ of each dNTP and $20 \mathrm{U}$ of RNAsin (Promega, Madison, WI, USA). An exogenous sample of reference RNA (50 ng of total rat liver RNA) was added as a source of internal standard for semi-quantitative Dp71 analysis.

The Dp71 transcript was analysed qualitatively by PCR amplification on $20 \mu \mathrm{l}$ of the cDNA sample, as previously described. ${ }^{16}$ PCR-amplified Dp71 cDNA was electrophoresed through $2 \%$ agarose gel and showed two bands of $2.1 \mathrm{~kb}$ and $1.8 \mathrm{~kb}$, respectively. The latter band results from al ternative splicing of exons 71 to 74 . For each patient, the full-length top band was extracted from the gel and purified using the gel Extraction kit (Qiagen, Hilden, Germany). Direct sequencing was performed using the ABI PRISM dye terminator cycle sequencing system (Perkin Elmer, Warrington, UK) (primers used for sequencing are available on request). The product from the sequencing reaction was analysed using a $4.25 \%$ denaturing polyacrylamide gel with a fluorescent DNA sequencer (ABI PRISM 377 DNA Sequencer, Perkin Elmer). Data were analysed automatically. RT-PCR was performed twice when a mutation was found.

For semi-quantitative Dp71 analysis, PCR was performed on $10 \mu \mathrm{l}$ of the cDNA sample with $50 \mathrm{pmol}$ of a forward primer identical to a sequence of the specific exon of Dp 71 (as above), and $50 \mathrm{pmol}$ of a reverse primer spanning nucleotides 9461-9482 in dystrophin cDNA, and on 50 pmol of two primers specific for rat liver L-type pyruvate kinase transcript (LPK1: AAGCAACGTAGCAGCATGGAA and LPK2: GGGTCAGTTGAGCCACACTCG). The cycling conditions used were $94^{\circ} \mathrm{C}(1 \mathrm{~min}), 58^{\circ} \mathrm{C}(1 \mathrm{~min})$, and $72^{\circ} \mathrm{C}(1 \mathrm{~min})$ for 17 cycles. The final PCR products were Southern blotted and hybridised using an internal 5' labelled oligonucleotide probe (CTTGCAGCCATGAGGGAACA) for Dp71 transcript and 5' labelled primer LPK1 for rat liver pyruvate kinase transcript.

Table 1 Summary of data on the 12 patients

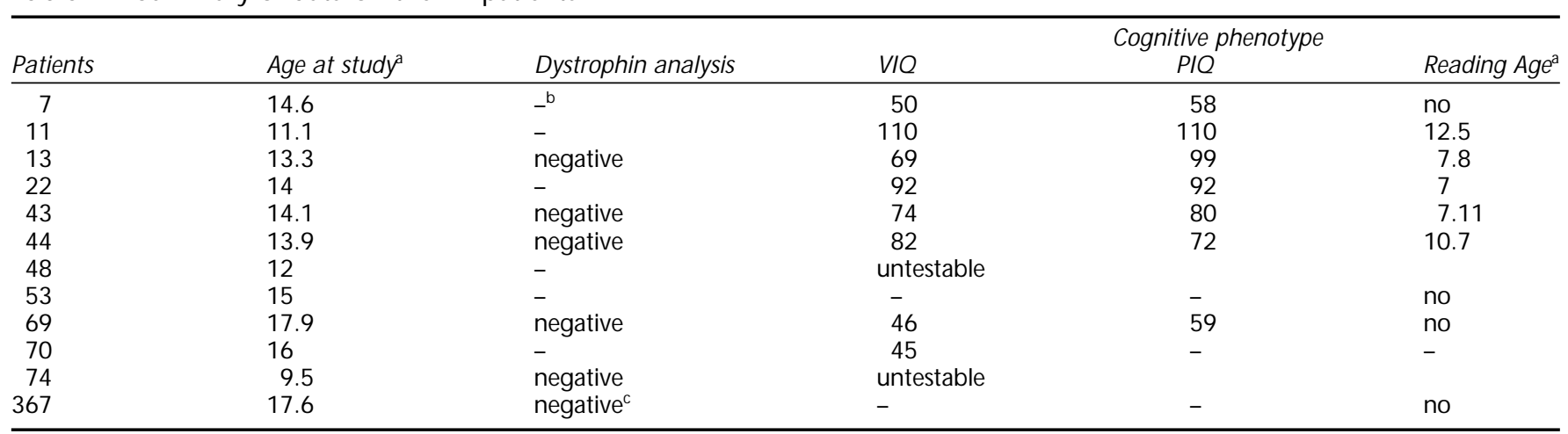

age in years, months; ${ }^{\text {b}}$ Data not available; 'Weak signal has been detected by western blot. 
Table 2 Mutations detected

\begin{tabular}{rlll}
\hline Patient & Exon & Nucleotide change & Amino acid change \\
\hline 74 & 66 & C $9776->T$ & Arg 3190->Stop \\
367 & 66 & C $9776->T$ & Arg 3190->Stop \\
69 & 66 & $9857+1 G->A$ & Thr 3188->>5s \\
53 & 69 & C 10241->T & Arg 3345->Stop \\
70 & 70 & C 10379->T & Arg 3391->Stop \\
\hline
\end{tabular}

fs: frameshift.

\section{Exon 66 analysis}

Exon 66 and its intronic boundaries were amplified and sequenced with specific primers (Leiden Muscular Dystrophy pages, web information) from $200 \mathrm{ng}$ of patient 69 genomic DNA.

\section{Results and discussion}

Table 2 summarizes the five sequence changes observed in the Dp71 coding sequence. All are translation termination mutations. Four of them are mutations of an arginine CGA codon corresponding to mutations at $\mathrm{CPG}$ sites, which are preferential sites for nonsense mutation $s^{17}$ and have al ready been described ${ }^{18,19}$ (Leiden Muscular Dystrophy pages, web information). The fifth is a mutation in the splice donor site of intron 66. Analysis of Dp71 mRNA from patient 69 by RT-PCR showed an abnormal pattern of migration (Figure1). Two slightly smaller products than the predicted bands were detected. Sequence analysis of the top band revealed a $85 \mathrm{bp}$ deletion corresponding to absence of exon 66 in the cDNA. This data was suggestive of an mRNA splicing defect. Exon 66 and its intronic boundaries were amplified from genomic DNA of patient 69 and controls. Direct sequencing showed a
$G$ to $A$ substitution at position +1 of the $5^{\prime}$ donor site in intron 66 . This mutation affects pre-mRNA maturation, leading to exon 66 skipping. The loss of exon 66 shifts the open reading frame, and thus introduces a termination codon at nucleotide position 9895 in exon 67. To the best of our knowledge this is a newly reported mutation.

These five chain terminating mutations are predicted to affect full-length muscle and brain type $427 \mathrm{kDa}$ dystrophins, either by truncation or by reduction of mRNA, or both, and should be considered causative of muscular disease. ${ }^{2}$ In addition, Dp71 might also be affected by truncation, probably leading to disruption of its function.

No mutation in Dp71 transcript was found in the five mildly or not retarded patients of this group (VIQ $\geq 70$, with delayed or correct reading acquisition). The five patients with a mutation were among the seven severely mentally retarded patients (VIQ $\leq 50$ and/or no reading acquisition or global mental deficiency). No mutation was found in Dp71 coding region in two patients ( 7 and 48 ) with severe cerebral dysfunction. Semi-quantitative analysis of Dp71 transcript by RT-PCR was performed to compare Dp71 transcript amount in these two patients with respect to patients with nonsense mutations and control (Figure2). As usually observed, ${ }^{20}$ a noticeable reduction in the level of Dp71 mRNA was observed in patients with a stop codon (patients 70 and 74). A similar reduction, indicative of an unstable mRNA and potentially in accordance with the psychometric phenotype, was observed for patient 7 . It is possible that a mutation, located either in Dp71 regulatory sequences or in the polyadenylation region of the transcript and which could explain the low level of mRNA, has not been detected in this patient. For patient 48, we found no

b
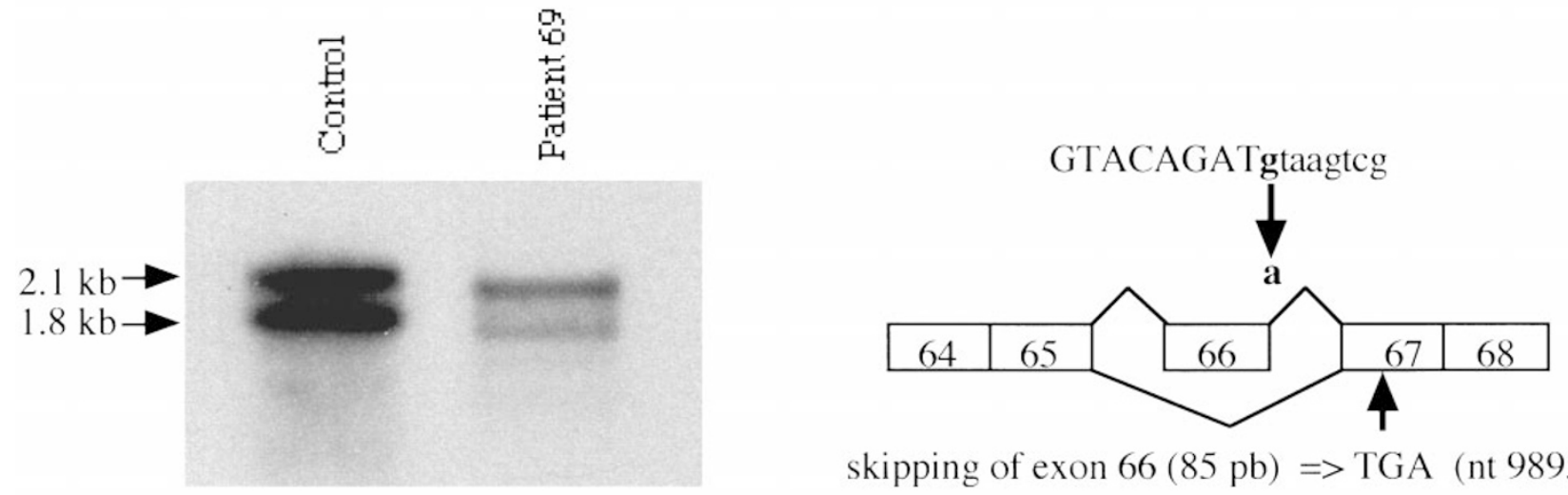

skipping of exon $66(85 \mathrm{pb})=>$ TGA (nt 9895)

Figure 1 Characterisation of the $9857+1 G->$ A mutation: a autoradiograph of Southern blot of the cDNA amplified products from a control and from patient 69. Dp 71 specific primer and a primer located in the $3^{\prime}$ untranslated region of the dystrophin mRNA (nucleotides 11519-11541) were used for amplification. Hybridisation was performed with a ${ }^{32} \mathrm{P}$ dCTP-labelled dystrophin cDNA probe covering exons53 to 65; b Splicing pattern in patient 69. Exons are shown as boxes. Partial sequence of exon 66 (capitals) and its intronic $5^{\prime}$ boundaries (lower case) amplified from the genomic DNA of patient 69 reveals a G - >A substitution at the junction exon 66-intron 66. 


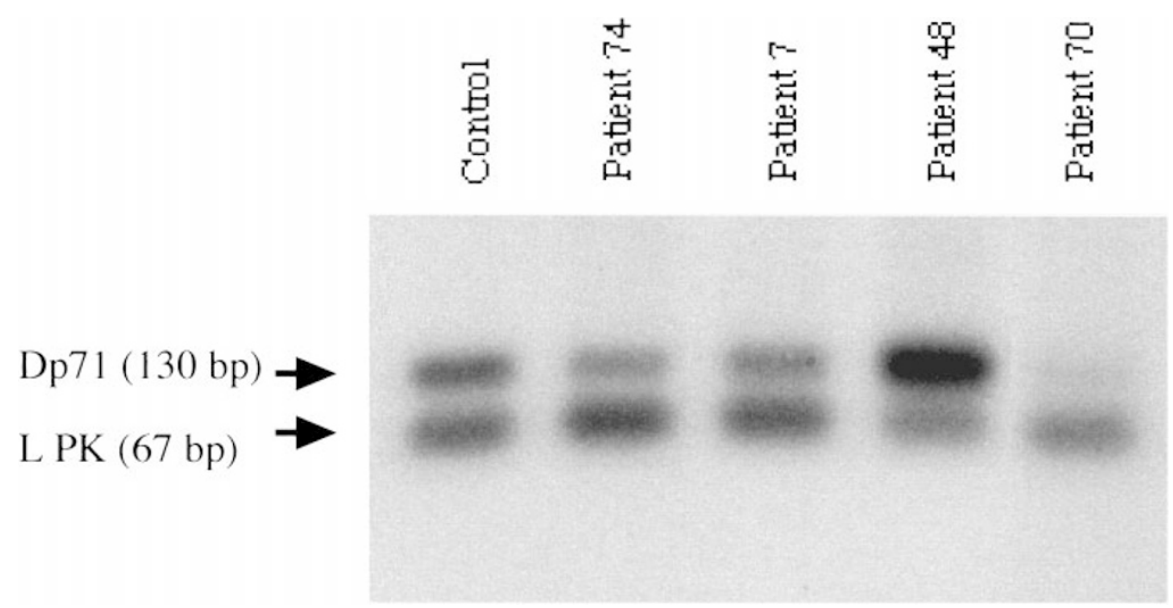

Figure 2 Expression of Dp71 transcript in DMD patients: autoradiograph of a Southern blot of the amplified cDNA products obtained after 17 cycles. The arrows indicate the size of the specific amplified fragments: $130 \mathrm{bp}$ for Dp71 transcript, $67 \mathrm{bp}$ for internal standard rat liver pyruvate-kinase transcript. Hybridisation was performed with an internal 5' radio-labelled oligonucleotide probe for Dp71 transcript and 5' radio-labelled LPK1 primer for rat liver pyruvate-kinase transcript.

evidence of unstable Dp71 transcript which could explain his intellectual disabilities. A Dp71 mutation, located outside the region pinpointed by the PCR primers and without any effect on Dp71 expression, could have been missed. However, a mutation outside the $\mathrm{Dp} 71$ region cannot be excluded in this case.

Finally, these results tend to confirm that mutations leading to premature translation termination in the Dp71 coding sequence are preferentially associated with severe mental retardation. Mutations in the distal part of the gene are likely to be associated with a loss of all isoforms expressed in the brain (including $427 \mathrm{kDa}$ brain isoforms) and that could explain why these patients have a particularly severe cognitive phenotype. Therefore, distal mutations seem to be more deleterious for brain function than proximal mutations which lead only to a loss of cerebral and cerebellar dystrophins. Among 19 point mutations reported to date in the Dp71 region in DMD patients with a known cognitive phenotype, 16 were indeed found in mentally retarded patients. ${ }^{14,15,18,19,21-28}$ Only three mutations were found in patients of normal intelligence. ${ }^{14,27,29}$ Two were located in exon 74 which is alternatively spliced in brain dystrophin isoforms, ${ }^{30}$ and one in exon 79 which corresponds to the $3^{1}$ untranslated region.

Although some of the mutations described here have al ready been reported, their associated cognitive phenotype has not been described. This study in fact adds five point mutations in exons62-79 associated with mental retardation.

Why the loss or alteration of Dp71 protein should be deleterious for cognitive function is unknown. Transgenic mice experiments have shown that Dp71 cannot replace the function of full-length dystrophin and correct the muscle defect, although it does restore the DAP complex. ${ }^{31}$ These results suggest that although Dp71 and dystrophin may interact with the same proteins they have distinct functions. Greenberg et $\mathrm{al}^{32}$ also demonstrated that mutant Dp71 deficient mice have a reduced level of DAP and conclude that Dp71 plays a role in the function or organisation of the DAP complex in the brain. It has been demonstrated that dystroglycan (a member of the DAP complex) and Dp 71 mRNAs, are co-located in some regions of the brain involved in certain cognitive processes. ${ }^{33,34}$ The early and gradually increased levels of Dp71 in the normal embryonic forebrain persisting to adulthood suggest its fundamental role during the development of the nervous system, ${ }^{35}$ indicating that lack of Dp71 could impair cognitive function.

In conclusion, this study is consistent with previous findings concerning the association between cognitive disabilities and the presence of mutation in the specific Dp71 region in DMD patients. The systematic screening of point mutations in the gene is hindered by the size and complexity of the dystrophin gene. However, direct detection of the mutation in probands provides the basis of accurate genetic counselling of DMD families. This study indeed demonstrates that severe cognitive impairment is good clinical evidence to suggest searching for point mutations in the Dp71 region in DMD patients with no detectable deletion or duplication.

\section{Acknowledgements}

The authors are grateful to the patients for their collaboration and to the physicians of medical centres for providing blood samples and clinical data from patients. We thank N Ronce and C Antar for their contribution to this work. S Briault and B Jauffrion for the lymphoblastoid cell lines and D Raine for her help in translation of the manuscript. This study was supported by grants from the Association Française contre les Myopathies (AFM). 


\section{References}

1 Koenig M, Hoffman EP, Bertelson CJ, Monaco AP, Feener C, Kunkel LM: Complete cloning of the Duchenne Muscular dystrophy (DMD) CDNA and preliminary genomic organization of the DMD gene in normal and affected individuals. Cell 1987; 50: 509-517.

2 Roberts RG, Gardner R, Bobrow M: Searching for the 1 in 2,400,000: a review of dystrophin gene point mutations. Hum Mut 1994; 4: 1-11.

3 Lidov HGW, Byers J, Watkins SC, Kunkel LM: Localization of dystrophin to postsynaptic regions of central nervous system cortical neurons. Nature 1990; 348: 725-727.

4 Gorecki DC, Monaco AP, Derry JMJ, Walker AP, Barnard EA, Barnard PJ: Expression of four alternative dystrophin transcripts in brain regions regulated by different promoters. Hum $\mathrm{Mol}$ Genet 1992; 1: 505-510.

5 Lidov HGW, Selig S, Kunkel LM: Dp140: a novel 140kDa CNS transcript from the dystrophin locus. Hum Mol Genet 1995; 4: 329-335.

6 Bar S, Barnea E, Levy Z, Neuman S, Yaffe D, Nudel U: A novel product of the Duchenne muscular dystrophy gene which greatly differs from the known isoforms in its structure and tissue distribution. Biochem J 1990; 272: 557-560.

7 Hugnot JP, Gilgenkrantz H, Vincent N et al: Distal transcript of the dystrophin gene initiated from an alternative first exon and encoding a 75-kDa protein widely distributed in non muscle tissues. Proc Natl Acad Sci USA 1992; 89: 7506-7510.

8 Ervasti JM, Campbell KP: Membrane organization of the dystrophin-glycoprotein complex. Cell 1991; 66: 1121-1131.

9 Billard C, Gillet P, Signoret JL et al: Cognitive functions in Duchenne muscular dystrophy: a reappraisal and comparison with spinal muscular atrophy. Neuromusc Disord 1992; 2: 371-378.

10 Rapaport D, Passos-Bueno MR, Brandao L, Love D, Vainzof M, Zatz $M$ : Apparent association of mental retardation and specific patterns of deletions screened with probes cf56a and cf23a in Duchenne muscular dystrophy. Am J Med Genet 1991; 39: 437-441.

11 Hodgson SV, Abbs S, Clark S et al: Correlation of clinical and deletion data in Duchenne and Becker muscular dystrophy, with special reference to mental ability. Neuromusc Disord 1992; 2: 269-276.

12 Bushby K, Appleton R, Anderson LVB, Welch JL, Kelly P, GardnerMedwin D: Deletion status and intellectual impairment in Duchenne muscular dystrophy. Dev Med Child Neurol 1995; 37: 260-269.

13 Bardoni A, Sironi M, Felisari G, Comi G, Bresolin N: Absence of brain Dp140 isoform and cognitive impairment in Becker muscular dystrophy. Lancet 1999; 353: 897-898.

14 Lenk U, Hanke R, Thiele H, Speer A: Point mutations at the carboxy terminus of the human dystrophin gene: Implications for an association with mental retardation in DMD patients. Hum Mol Genet 1993; 2: 1877-1881.

15 Tuffery S, Lenk U, Roberts RG, Coubes C, Demaille J, Claustres M: Protein truncation test: Analysis of two novel point mutations at the carboxy-terminus of the human dystrophin gene associated with mental retardation. Hum Mut 1995; 6: 126-135.

16 Moizard MP, Billard C, Toutain A, Berret F, Marmin N, Moraine C: Are Dp71 and Dp 140 brain dystrophin isoforms related to cognitive impairment in Duchenne muscular dystrophy? Am J Med Genet 1998; 80: 32-41.

17 Akalin N, Zietkiewicz E, Makalowski W, Labuda D: Are CpG sites mutation hot spots in the dystrophin gene? Hum M ol Genet 1994; 3: 1425-1426.
18 Barbieri AM, Soriani N, Ferlini A, Michelato A, Ferrari M, Carrera $P$ : Seven novel additional small mutations and a new alternative splicing in the human dystrophin gene detected by heteroduplex analysis and restricted RT-PCR heteroduplex analysis of illegitimate transcripts. Eur J Hum Genet 1996; 4: 183-187.

19 Tuffery S, Chambert S, Bareil C et al: Mutation analysis of the dystrophin gene in Southern French DMD or BMD families: from Southern blot to protein truncation test. Hum Genet 1998; 102: 334-342.

20 Mclntosh I, Hamosh A, Dietz $\mathrm{H}$ : Nonsense mutations and diminished mRNA levels. Nat Genet 1993; 4: 219.

21 Roberts RG, Bobrow M, Bentley DR: Point mutations in the dystrophin gene. Proc Natl Acad Sci USA 1992; 89: 2331-2335.

22 Lenk U, Oexle K, Voit T et al: A cystein 3340 substitution in the dystroglycan-binding domain of dystrophin associated with Duchenne muscular dystrophy, mental retardation and absence of the ERG b-wave. Hum Mol Genet 1996; 5: 973-975.

23 Tsukamoto H, Inui K, Matsuoka T, Yanagihara I, Fukushima H, Okada S: One base deletion in the cysteine-rich domain of the dystrophin gene in Duchenne muscular dystrophy patients. Hum Mol Genet 1994; 3: 995-996.

24 Comi GP, Ciafaloni E, Rohan de Silva $\mathrm{HA}$ et al: $\mathrm{A} \mathrm{G}^{+1}->\mathrm{A}$ transversion at the 5 ' splice site of intron 69 of the dytrophin gene causing the absence of peripheral nerve Dp116 and severe clinical involvement in a DMD patient. Hum Mol Genet 1995; 4: 2171-2174.

25 Gardner RJ, Bobrow M, Roberts RG: The identification of point mutations in Duchenne muscular dystrophy patients by using reverse-transcription PCR and the protein truncation test. Am J Hum Genet 1995; 57: 311-320.

26 Roest PAM, Bout M, van der Tuijen AC et al: Splicing mutations in DMD/BMD detected by RT-PCR/PTT: detection of a 19aa insertion in the cysteine rich domain of dystrophin compatible with BMD. J Med Genet 1996; 33: 935-939.

27 Lasa A, Gallano P, Baiget M: Three novel point mutations in the dystrophin gene in DMD patients. Hum Mut 1997; 9: 473-474.

28 Cau M, Cao A, Loi D et al: Two novel mutations (10410 T-> G; $10296 \mathrm{delC})$ at carboxy-terminus of the dystrophin gene associated with mental retardation. Hum Mut 1998; 12: 70-73.

29 Nigro V, Nigro G, Esposito MG et al: Novel small mutations along the DMD/BMD gene associated with different phenotypes. Hum Mol Genet 1994; 3: 1907-1908.

30 Bies RD, Phelps SF, Cortez MD, Roberts R, Caskey CT, Chamberlain JS: Human and murine dystrophin mRNA transcripts are differentially expressed during skeletal muscle, heart, and brain development. Nucleic Acids Res 1992; 20: 1725-1731.

31 Greenberg DS, Sunada Y, Campbell KP, Yaffe D, Nudel U: Exogenous Dp71 restores the levels of dystrophin associated proteins but does not alleviate muscle damage in mdx mice. Nat Genet 1994; 8: 340-344.

32 Greenberg DS, Schatz Y, Levy Z, Pizzo P, Yaffe D, Nudel U: Reduced levels of dystrophin associated proteins in the brains of mice deficient for Dp71. Hum Mol Genet 1996; 5: 1299-1303.

33 Gorecki DC, Derry JMJ, Barnard EA: Dystroglycan: brain localisation and chromosome mapping in the mouse. Hum Mol Genet 1994; 3: 1589-1597.

34 Gorecki DC, Barnard E: Specific expression of G-dystrophin (Dp71) in the brain. NeuroReport 1995; 6: 893-896.

35 Sarig R, Mezger-Lallemand V, Gitelman I et al: Targeted inactivation of Dp71, the major non-muscle product of the DMD gene: differential activity of the Dp71 promoter during development. Hum Mol Genet 1999; 8: 1-10. 